\title{
Compostos bioativos e atividade antirradicalar em farinhas de bagaço de uvas de diferentes cultivares desidratadas em liofilizador e em estufa
}

\author{
Bioactive compounds and antiradical activity in grape pomace flours from different \\ cultivars dehydrated in a freeze dryer and in an oven
}

\author{
Gabriela Datsch Bennemann 1* (), Renato Vasconcelos Botelho², Yohandra Reyes Torres ${ }^{3}$, \\ Luciana Alves Camargo ${ }^{4}$, Najeh Maissar Khalil' ${ }^{2}$, Tatiane Luiza Cadorin Oldoni ${ }^{5}$, Diego Henrique da Silva ${ }^{5}$ \\ ${ }^{1}$ Universidade Estadual do Centro Oeste (Unicentro), Departamento de Nutrição, Guarapuava/PR - Brasil \\ 2 Universidade Estadual do Centro Oeste (Unicentro), Departamento de Agronomia, Guarapuava/PR - Brasil \\ ${ }^{3}$ Universidade Estadual do Centro Oeste (Unicentro), Departamento de Química, Guarapuava/PR - Brasil \\ ${ }^{4}$ Universidade Estadual do Centro Oeste (Unicentro), Departamento de Farmácia, Guarapuava/PR - Brasil \\ ${ }_{5}^{5}$ Universidade Federal Tecnológica do Paraná (UTFPR), Departamento de Química, Pato Branco/PR - Brasil
}

\section{*Corresponding Author}

Gabriela Datsch Bennemann, Universidade Estadual do Centro Oeste (Unicentro), Departamento de Nutrição, Rua Simeão Varela de Sá, 3, Vila Carli, CEP: 85040-080, Guarapuava/PR - Brasil, e-mail: gabibennemann@gmail.com

Cite as: Bioactive compounds and antiradical activity in grape pomace flours from different cultivars dehydrated in a freeze dryer and in an oven. Braz. J. Food Technol., v. 21, e2017205, 2018

\section{Resumo}

Este estudo objetivou caracterizar compostos bioativos e atividade antirradicalar em farinhas de bagaços de uvas das cultivares Cabernet Sauvignon, Merlot, Sauvignon Blanc e Sangiovese (Vitis vinifera), desidratados em estufa de circulação de ar a $45^{\circ} \mathrm{C}$ ou liofilizador a vácuo. Foram analisados compostos fenólicos totais e individuais, antocianinas monoméricas e atividade antirradicalar pelos métodos $\mathrm{ABTS}$ e $\mathrm{HOCl}$. A perda total de antocianinas monoméricas foi maior para as cultivares Cabernet Sauvignon e Sangiovese, pelo método de desidratação em estufa. Todas as cultivares liofilizadas preservaram índices estatisticamente superiores de compostos fenólicos totais, com superioridade para a cultivar Sauvignon Blanc. Na análise individual destes compostos, as amostras liofilizadas tiveram maiores teores médios de todos os flavonoides (epicatequina, catequina, quercetina, ácido vanílico, rutina), enquanto que os ácidos gálico, cafeico e ferrúlico, aparentemente, não foram influenciados pelo método de secagem. Todas as amostras desidratadas em estufa tiveram menor capacidade de inibição dos radicais livres em relação às amostras liofilizadas, por ambos os métodos de IC50. O método ABTS apresentou os melhores resultados para as amostras liofilizadas, ou seja, quantidades menores das farinhas foram capazes de inibir em pelo menos $50 \%$ a atividade dos radicais livres ABTS e HOCl, com destaque para a cultivar Merlot.

Palavras-chave: Compostos fenólicos; Resíduo vinícola, Vinificação, Alimento funcional; Nutrição; Atividade antirradicalar.

\section{Abstract}

This study aimed to characterize the bioactive compounds and antiradical activity in grape pomace flours from the cultivars Cabernet Sauvignon, Merlot, Sauvignon Blanc and Sangiovese (Vitis vinifera), dehydrated either in an air circulation oven at $45^{\circ} \mathrm{C}$ or a vacuum freeze dryer. The total and individual phenolic compounds, monomeric anthocyanins and antiradical activity were analysed by the ABTS and $\mathrm{HOCl}$ methods. The total loss of monomeric anthocyanins was higher for the cultivars Cabernet Sauvignon and Sangiovese dehydrated in the air circulation oven. All the freeze dried cultivars preserved a statistically higher index for the total phenolic compounds, the highest being the cultivar Sauvignon Blanc. In the individual analysis of these compounds the freeze dried samples showed higher average levels of all the flavonoids (epicatechin, catechin, quercetin, vanillic acid, rutin), whereas gallic, caffeic and ferulic acids were apparently not influenced by the drying method. All the samples dehydrated in the oven showed less free radical inhibition capacity than the freeze dried samples as determined by both IC50 methods. The ABTS method presented the best results for the freeze dried samples, that is, smaller amounts of the flours were able to inhibit at least $50 \%$ of the ABTS and $\mathrm{HOCl}$ free radical activities, with emphasis on the Merlot cultivar.

Keywords: Phenolic compounds; Winery waste; Winemaking; Functional food; Nutrition; Antiradical activity. 


\section{Introdução}

A vitivinicultura é uma atividade de importância econômica mundial, com uma produção anual estimada em 60 milhões de toneladas. Cerca de $80 \%$ deste montante é destinado à elaboração de vinhos e derivados, o que resulta anualmente em cerca de 13 milhões de toneladas de resíduos (OIV, 2016). A produção brasileira é bastante expressiva, com aproximadamente 1,3 milhão de toneladas de uvas destinadas à produção de vinho e para o consumo in natura, com destaque para os Estados da Região Sul (BRASIL, 2016).

Botanicamente, a uva pode ser definida como um fruto da videira, pertencente à família das vitáceas e ao gênero Vitis, com as seguintes espécies: Vitis vinifera, Vitis labrusca, Vitis rupestris, Vitis aestivalis, Vitis riparia, Vitis cinerea, Vitis rotundifolia, entre outras (POUDEL et al., 2008). Deste modo, esta ampla diversidade genética ocasiona diferenças na composição química das uvas, o que permite uma seleção, tanto para o seu uso industrial como para o consumo in natura (NARDUZZI et al., 2015).

Abe et al. (2007) desenvolveram estudo com farinhas de bagaços de sete cultivares de uvas produzidas em Minas Gerais, mais especificamente das espécies Vitis labrusca (cv. Niágara rosada, nos porta-enxertos IAC 766 e 196-17, e cv. Folha de Figo, nos porta-enxertos 420A e 196-17) e três cultivares da espécie Vitis vinifera (cv. Syrah - porta-enxerto 3309 C, cv. Merlot - porta-enxerto 1103 P e cv. Moscato Embrapa - porta-enxerto IAC 766), safra 2005/2006. As antocianinas monoméricas foram os compostos encontrados em maior porcentagem nas cultivares Folha de Figo, nos porta-enxertos 420A e 196-17, Syrah e Merlot. A cultivar Moscato Embrapa, de bagas brancas, foi a que se destacou por apresentar menor quantidade de fenólicos totais. Esta cultivar demonstrou ausência de antocianinas monoméricas e também não apresentou nenhum outro composto em quantidades relevantes. Os autores afirmam que o perfil de compostos fenólicos nas cultivares avaliadas não dependeu necessariamente da espécie, sendo mais variável de acordo com a cultivar.

Os resíduos vitivinícolas apresentam alto conteúdo de fibras, flavonoides, antocianinas, substâncias aromáticas, ácidos, taninos e micro-organismos responsáveis pela fermentação do mosto (KARLING et al., 2017). Em grande parte, este material é descartado e pode provocar impactos ambientais e um desperdício de matéria orgânica (MELO et al., 2011). Na literatura científica, há relatos de inclusão de resíduos agroindustriais, tais como subprodutos do processamento de polpa de fruta e farinha de bagaço de uva em pães (PAZ et al., 2015), biscoitos e cookies (ABUD; NARAIN, 2009; PIOVESANA et al., 2013; KARNOPP et al., 2015) e snacks extrusados (BENDER et al., 2016).

Os compostos fenólicos são definidos como o maior grupo de antioxidantes naturais, com cerca de
8.000 compostos diferentes. Estão largamente distribuídos nos alimentos vegetais, sendo metabólitos secundários destes e considerados fundamentais para o desenvolvimento adequado da planta e a defesa contra injúrias ambientais e processos infecciosos (ABDRABBA; HUSSEIN, 2015). Apresentam ação antioxidante contra radicais livres e, segundo algumas evidências, supõe-se que os compostos fenólicos possuem efeitos em cardiopatias, principalmente na redução do infarto (SOARES et al., 2008; DAVIDOV-PARDO et al., 2011).

Os flavonoides de maior relevância nas uvas e seus derivados são as antocianinas, as quais constituem a maior parte dos compostos fenólicos presentes, e encontram-se largamente distribuídos na natureza (MUÑOZ-ESPADA et al., 2004). As antocianinas são responsáveis pela coloração arroxeada das uvas, ocorrendo naturalmente como glicosídeos dissolvidos no fluido celular, particularmente no tecido epidérmico. Sua concentração nos alimentos é condicionada por diversos fatores agronômicos, como cultivar, clima, tipo de solo e práticas agrícolas (FALCÃO et al., 2007; SEGADE et al., 2008). As antocianinas também atuam como filtro das radiações ultravioletas das folhas e, quando consumidas na alimentação humana, desempenham diversas propriedades farmacológicas, como atividade antioxidante, prevenção de enfermidades cardiovasculares e neuronais, câncer e diabetes (MANACH et al., 2004).

A quantidade de água livre presente nos alimentos é uma das principais causas dos processos de deterioração. Desta forma, a desidratação de alimentos tem sido proposta como um importante método de conservação, cujo objetivo principal é a remoção parcial de água dos alimentos. A desidratação em estufa, por exemplo, apresenta como vantagens principais o baixo custo e a obtenção de produtos com segurança microbiológica satisfatória (GOMEZCACERES et al., 2013). Embora ainda sejam restritos os estudos que avaliam o efeito do método de desidratação sobre os compostos bioativos de alimentos, algumas pesquisas apontam que a temperatura de secagem também possa ter um efeito considerável sobre tais compostos (HAMAMA; NAWAR, 1991; LARRAURI et al., 1997; MARFIL et al., 2008).

Processos de desidratação de alimentos que não empregam o calor como mecanismo de remoção de água, como a liofilização, são eficientes na conservação dos compostos bioativos de alimentos. Esta metodologia é particularmente interessante para a preservação dos polifenóis, os quais, segundo Malacrida e Motta (2005), são sensíveis à elevação da temperatura.

Este estudo objetivou o desenvolvimento de farinhas a partir de resíduos de produção vinícola pelos métodos de desidratação em estufa e liofilização, verificando se estes processos influenciam nos índices de compostos bioativos e atividade antirradicalar nestes produtos provenientes de diferentes cultivares de uva. 


\section{Material e métodos}

Para as análises de comparação entre os processos de desidratação em estufa e liofilizador, utilizaram-se as cultivares de uva Merlot, Cabernet Sauvignon, Sangiovese e Sauvignon Blanc, colhidas na safra 2015-2016, provenientes da Vinícola Abreu \& Garcia, situada na cidade de Campo Belo do Sul, Santa Catarina, a 1.017 m de altitude, latitude $27^{\circ} 53^{\prime} 57^{\prime \prime}$ S e longitude $50^{\circ} 45^{\prime} 39^{\prime \prime} \mathrm{O}$.

Foram coletadas seis amostras de bagaço de uva, de cada cultivar, de aproximadamente 500 gramas, retiradas por ocasião da descuba, para serem armazenadas sob congelamento a $-18{ }^{\circ} \mathrm{C}$. A coleta deste material para transporte até o local do processamento da farinha só ocorreu após congelamento completo dos resíduos.

As amostras para secagem em estufa foram acondicionadas em bandejas de papel alumínio e mantidas em estufa microprocessada com circulação e renovação de ar modelo SL 102 (Solab, Piracicaba, São Paulo, Brasil) a $45^{\circ} \mathrm{C}$, até atingir a umidade mais baixa que o índice de $14 \%$ p/p, indicada para a produção de farinhas de resíduos de uvas (APHA, 2001). Posteriormente, permaneceram em temperatura ambiente $\left(22^{\circ} \mathrm{C}\right)$ até total resfriamento.

Para o preparo das farinhas pelo método de desidratação em liofilizador, as amostras de bagaços previamente congeladas a $-18{ }^{\circ} \mathrm{C}$ foram desidratadas em liofilizador de bancada modelo LD 1500 (Terroni, São Carlos, São Paulo, Brasil).

Após a secagem, todas as amostras foram submetidas à tamisação em conjunto de peneiras granulométricas do tipo tamis, com aberturas de malhas de 10, 14 e 18 Tyler (Bertel, Caieiras, São Paulo, Brasil). Em seguida, as cascas e sementes foram trituradas em moinho rotor tipo ciclone/Willey, modelo MA1340 (Marconi, Piracicaba, São Paulo, Brasil), até a obtenção da farinha de resíduos de uvas. Este material foi novamente submetido à tamisação em peneiras com aberturas de malhas de 28 Tyler. As alíquotas de farinhas empregadas nas análises foram acondicionadas em frascos de vidro âmbar e armazenadas em ultrafreezer a $-80{ }^{\circ} \mathrm{C}$ até o momento das análises.

As amostras submetidas às análises de compostos fenólicos totais, antocianinas monoméricas e compostos fenólicos individuais, nas farinhas, foram extraídas pelo uso do solvente etanol. Utilizaram-se 2,5 $\mathrm{g}$ de farinha acrescidos de $15 \mathrm{~mL}$ de solvente etanol $80 \%$. Para a determinação da atividade antirradicalar pelos métodos ABTS e $\mathrm{HOCl}$, foram utilizados inicialmente $10 \mathrm{mg}$ de farinha diluídos em $1 \mathrm{~mL}$ de etanol 80\% (v/v).

Para a determinação do conteúdo total de antocianinas monoméricas, foram utilizados $200 \mu \mathrm{L}$ dos extratos aquosos, seguindo-se o método de diferença de pH (GIUSTI; WROLSTAD, 2001). Foram elaboradas duas soluções tampão, uma de cloreto de potássio (0,025M) de $\mathrm{pH}$ 1,0 e outra de acetato de sódio $(0,4 \mathrm{M})$ de pH 4,5. Foram feitas leituras a $520 \mathrm{~nm}$ e $700 \mathrm{~nm}$, tanto no tampão de $\mathrm{pH}$ 1,0 quanto de pH 4,5 (espectrofotômetro UV-VIS, modelo UV-1800, Shimadzu, Barueri, São Paulo, Brasil). A leitura a $700 \mathrm{~nm}$ foi realizada para descontar a turbidez da amostra e os resultados foram apresentados em equivalentes de malvidina 3-glicosídeo.

A análise do teor de compostos fenólicos totais foi realizada de acordo com o método espectrofotométrico de Folin-Ciocateau descrito por Singleton e Rossi (1965), utilizando ácido gálico como padrão. O procedimento foi realizado em triplicata, transferindo-se uma alíquota de $0,1 \mathrm{~mL}$ do extrato em etanol $80 \%$ (v/v) para um tubo de ensaio e adicionando-se $0,5 \mathrm{~mL}$ do reagente Folin-Ciocalteau $10 \mathrm{~g} \mathrm{~L}^{-1}$. A absorbância foi medida em espectrofotômetro UV-VIS a 740 nm (UV-1800, Shimadzu, Barueri, São Paulo, Brasil).

Os compostos fenólicos individualizados foram analisados pelo método de cromatografia líquida de alta eficiência (HPLC), de acordo com metodologia adaptada por Silva (2016). O procedimento foi realizado em Tubo Falcon, utilizando uma alíquota de 2,5 mL dos extratos de farinha de bagaço de uva, extraído duas vezes com $5 \mathrm{~mL}$ de etanol 80\% (v/v) sob agitação em vórtex. As fases orgânicas foram combinadas e centrifugadas por 10 min a 3.000 rpm, para garantir uma melhor separação e retirada total da fase aquosa. Após centrifugação, o solvente da fase orgânica foi evaporado em evaporador e concentrador a vácuo, em temperatura ambiente. O resíduo obtido foi redissolvido em 1,2 mL de solução acetonitrila:água (40:60 v/v). A análise foi realizada utilizando-se um sistema LC 920 (Varian Inc., Walnut Creek, CA, EUA). O software Galaxie foi usado para controlar o amostrador automático, as configurações de gradiente, o monitor Diode Array (DAD) e a aquisição de dados. Uma coluna C18 RP (250 mm × 4,6 mm, 5 um) (Eclipse Plus, Agilent Technologies, Wilmington, DE, EUA), mantida a $30{ }^{\circ} \mathrm{C}$, foi empregada na análise. Foram injetados volumes de $10 \mu \mathrm{L}$ de extratos a uma concentração de $80 \mathrm{~g} \mathrm{~L}^{-1}$. A fase móvel consistiu de uma mistura de gradiente de solvente A (solução aquosa de ácido acético a $2 \%$ ) e solvente $B$ (acetonitrilo a $40 \%$ acidificado com solução aquosa a $2 \%$ de ácido acético), a uma taxa de fluxo de $1 \mathrm{~mL}$. O gradiente foi iniciado com $5 \%$ de solvente B e ajustado para $20 \%$ a $2 \mathrm{~min} ; 25 \%$ de B, a $15 \mathrm{~min} ; 85 \%$ de B, a $25 \mathrm{~min}$, mantido durante 5 minutos; $20 \%$ de B, a 33 min; $5 \%$ de B, em 36 min, até $44 \mathrm{~min}$, como etapa de condicionamento. As áreas de pico foram determinadas a $280 \mathrm{~nm}$ para ácido gálico, ácido vanílico e flavanóis: catequina e epicatequina; 300 nm para ácido cumárico, ácido salicílico e resveratrol; 320 nm para ácido cafeico e ferúlico, e 360 nm para flavonoides: rutina, quercetina e miricetina.

Para as análises da atividade antirradicalar das amostras utilizou-se o extrato hidroalcoólico. Para o método 
do radical livre ABTS (CAMARGO et al., 2016), trabalhou-se com $10 \mathrm{mg}$ de amostra em $200 \mu \mathrm{L}$ de solução estoque de ABTS e $200 \mathrm{~mL}$ de tampão (10 $\mathrm{mg} \mathrm{mL}^{-1}$ ). A partir desta solução estoque, realizaram-se diluições de $200 \mu \mathrm{L}$ de solução estoque e $200 \mathrm{~mL}$ de tampão, até a proporção de 1:32 (v/v). Estas amostras foram submetidas ao radical livre, para avaliar até qual diluição estas eram capazes de apresentar atividade antirradicalar representativa.

Inicialmente, uma mistura aquosa de ABTS $\left(7 \mathrm{mmol} \mathrm{L}^{-1}\right)$ e persulfato de potássio $\left(2,45 \mathrm{mmol} \mathrm{L}^{-1}\right)$ foi incubada à temperatura ambiente e ao abrigo da luz, por 12 horas. A solução formada de ABTS • foi diluída em tampão fosfato salina (PBS) $50 \mathrm{mmol} \mathrm{L}^{-1}$, pH 7,4, a uma absorbância de 0,70 (734 nm). Soluções de diferentes concentrações dos extratos foram incubadas com o ABTS*+ por 30 minutos, à temperatura ambiente, e a redução do ABTS*+ pelos extratos foi observada a $734 \mathrm{~nm}$ em espectrofotômetro (modelo UV-1800, Shimadzu, Barueri, São Paulo, Brasil) (CAMARGO et al., 2016).

Para a determinação da atividade antirradicalar pelo método $\mathrm{HOCl}$ (CAMARGO et al., 2016), se utilizou a solução de ácido hipocloroso $\left(\mathrm{HOCl} 1 \mathrm{mmol} \mathrm{L}^{-1}\right)$ preparada a partir de uma solução de $\mathrm{NaOCl}$ em $\mathrm{NaOH} 50 \mathrm{mmol} \mathrm{L}^{-1}$. A concentração da solução de HOCl foi verificada espectrofotometricamente usando o coeficiente de extinção molar $350 \mathrm{~cm}^{-1} \mathrm{M}^{-1} \mathrm{em} 292 \mathrm{~nm}$ (espectrofotômetro modelo UV-1800, Shimadzu, Barueri, São Paulo, Brasil). A solução de 3,3',5,5'-tetramethylbenzidine (TMB) a $0,014 \mathrm{~mol} \mathrm{~L}^{-1}$ foi preparada se dissolvendo $0,017 \mathrm{~g}$ de TMB em 2,5 mL de dimetilformamida, 2,45 mL de ácido acético $0,8 \mathrm{~mol} \mathrm{~L}^{-1}$ e $0,05 \mathrm{~mL}$ de $\mathrm{KI} 0,01 \mathrm{~mol} \mathrm{~L}^{-1}$. A reação ocorreu em PBS $50 \mathrm{mmol} \mathrm{mL}^{-1}$, pH 7,4, contendo diferentes concentrações da amostra, adicionado de $75 \mu \mathrm{mol} . \mathrm{L}^{-1}$ de $\mathrm{HOCl}$, com incubação de 15 minutos, ao abrigo da luz e em temperatura ambiente de aproximadamente $17^{\circ} \mathrm{C}$. O HOCl remanescente foi revelado pela adição de uma solução de TMB $\left(0,014\right.$ mol. $\left.L^{-1}\right)$, cujo produto de oxidação possui absorbância máxima em 652 nm (CAMARGO et al., 2016).

Os resultados foram submetidos à análise de variância (ANOVA), com o teste de post-hoc SNK para comparação das médias (pacote estatístico SISVAR 5.6) (FERREIRA, 2011). Para as análises de cromatografia, foi realizada a análise de componentes principais (PCA) por meio do software MINITAB R14 (TABACHNICK; FIDELL, 2007).

\section{Resultados e discussão}

\subsection{Conteúdo de antocianinas monoméricas e compostos fenólicos totais em farinhas de bagaços de uva desidratadas em estufa e liofilizador}

As farinhas apresentaram teores de antocianinas

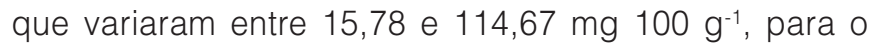

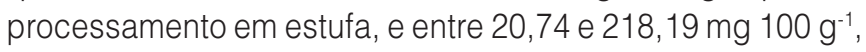
para o material liofilizado. As farinhas provenientes de bagaço da uva Cabernet Sauvignon apresentaram os maiores teores de antocianinas monoméricas, seguida pela cultivar Sangiovese, enquanto que os menores valores foram verificados para as cultivares Merlot e Sauvignon Blanc (Figura 1A). As amostras desidratadas em estufa das cultivares Cabernet Sauvignon e Sangiovese apresentaram teores significativamente inferiores de antocianinas monoméricas quando comparados aos valores obtidos pelo processo de liofilização. Para as cultivares Merlot e Sauvignon Blanc, não houve diferenças entre os processos de desidratação.

Verifica-se que tanto a cultivar quanto o método de secagem influenciaram os teores de antocianinas, mas a cultivar da uva foi mais importante em determinar os teores médios destes compostos (Figura 1A). De forma semelhante, Abe et al. (2007), em estudo com farinhas de bagaços de uvas de sete cultivares distintas das espécies Vitis vinifera e Vitis labrusca, identificaram conteúdos de antocianinas variando entre $12,8 \pm 0,1$ e $248 \pm 24 \mathrm{mg}$ equivalentes de malvidina 3-glicosídeo $100 \mathrm{~g}^{-1}$, com índices estatisticamente distintos das antocianinas malvidina, delfinidina e peonidina, entre as cultivares Folha de Figo, Syrah e Merlot.

Os índices de antocianinas nos bagaços de uvas são expressos por mais de cinquenta tipos destes compostos,
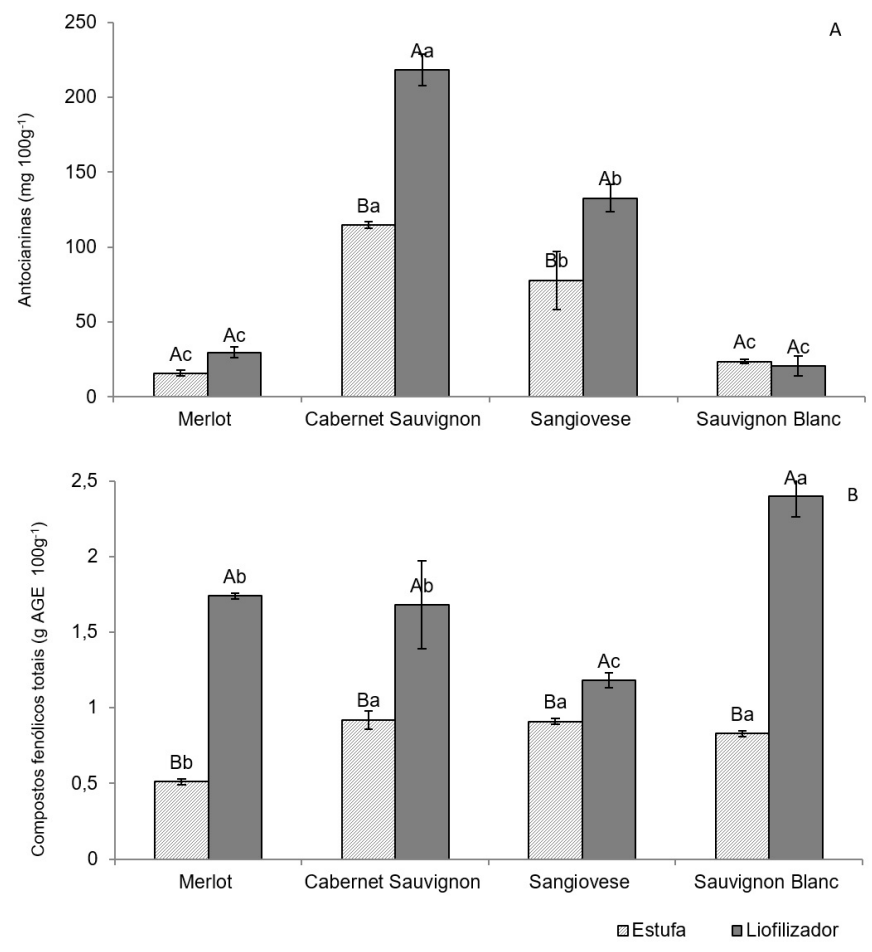

Figura 1. Conteúdo de antocianinas (A) e compostos fenólicos totais (B) em farinhas de bagaço de uvas desidratadas em estufa e liofilizador). Médias seguidas pela mesma letra (maiúsculas para tipo de processo e minúsculas para cultivares) não diferem entre si pelo teste de Student Neuman-Keuls $(p \leq 0,05)$. Barras verticais representam o desvio padrão $(n=3)$. 
sendo estas as principais: cianidina, peonidina, delfinidina, petunidina e malvidina (OLIVEIRA et al., 2015). A degradação térmica destas antocianinas forma produtos de coloração marrom, especialmente na presença de oxigênio, que, juntamente com outros agentes oxidantes presentes nos alimentos, pode acelerar sua degradação (JACKMAN; SMITH, 1992). Mourtzinos et al. (2008), ao estudarem a estabilidade térmica de antocianinas, observaram um pico exotérmico em torno de $220^{\circ} \mathrm{C}$ relacionado à oxidação deste pigmento. Isto poderia justificar as menores perdas deste composto, uma vez que as temperaturas utilizadas não atingiram este limiar térmico.

A liofilização proporcionou a manutenção de maiores conteúdos de compostos fenólicos totais para todas as variedades analisadas (Figura 1B). Em relação às cultivares, verificou-se que a farinha proveniente da cv. Sauvignon Blanc pelo método de liofilização apresentou o maior conteúdo de compostos fenólicos. Para as amostras secas em estufa, a farinha da cv. Merlot apresentou o menor teor de polifenóis (Figura 1B).

Neste estudo, foram observadas perdas maiores de compostos fenólicos totais, em comparação às antocianinas, no processo de desidratação em estufa em relação à liofilização. A secagem em estufa utilizou uma temperatura relativamente baixa $\left(45^{\circ} \mathrm{C}\right)$, porém por um longo período (quatro dias). O método de liofilização é particularmente benéfico para a preservação dos polifenóis, uma vez que estes compostos são sensíveis à temperatura (CORREA-BETANZO et al., 2015). A extração a quente é capaz de converter compostos fenólicos insolúveis em solúveis (CATANEO et al., 2008). Porém, este processo não consegue romper as ligações covalentes dos compostos fenólicos, acarretando perdas na quantidade destes compostos, principalmente devido à degradação de antocianinas (MALACRIDA; MOTTA, 2005).

\subsection{Conteúdos de compostos fenólicos individuais para as farinhas de bagaço de uva desidratadas em estufa e em liofilizador}

A Figura 2 apresenta a projeção dos componentes principais (CPs) dos conteúdos de compostos fenólicos para as amostras desidratadas em estufa e liofilizador. No gráfico formado pelas CP-1 vs. CP-2, que juntas explicam $65 \%$ da variabilidade dos dados em relação ao método de secagem, observa-se que praticamente todas as amostras liofilizadas (independentemente da cultivar de uva) situam-se no eixo negativo da CP-1 (exceção da cultivar Merlot liofilizada e uma repetição da cv. Cabernet Sauvignon liofilizada). Por outro lado, quase todas as amostras submetidas à secagem em estufa encontram-se no eixo positivo da CP-1, com exceção de duas repetições da cultivar Sauvignon Blanc secas em estufa. Essa disposição sugere que o método de secagem influencia os teores de compostos fenólicos muito mais do que a característica do resíduo de cada cultivar de uva. Também se verificou que amostras de farinhas da mesma cultivar formam clusters distintos, dependendo do método de secagem (Figura 2). Isso sugere que o método de secagem foi bastante preponderante na composição dos teores dos compostos fenólicos das amostras.

Observou-se também que, de maneira geral, as amostras liofilizadas tiveram maiores teores médios de todos os flavonoides (epicatequina, catequina, quercetina, ácido vanílico, rutina) (Figura 2). Os teores dos ácidos gálico, cafeico e ferrúlico, aparentemente, não foram influenciados pelo método de secagem. Além disso, teores representativos de ácido cafeico foram identificados somente na cultivar Merlot (desidratadas pelos dois métodos), porém sendo maiores nas amostras desidratadas em estufa

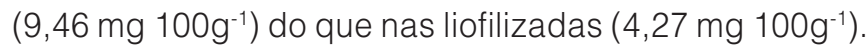
Isso deve estar relacionado a termolabilidade e volatilidade de cada composto. Observa-se o mesmo na análise hierárquica, conforme a Figura 3.
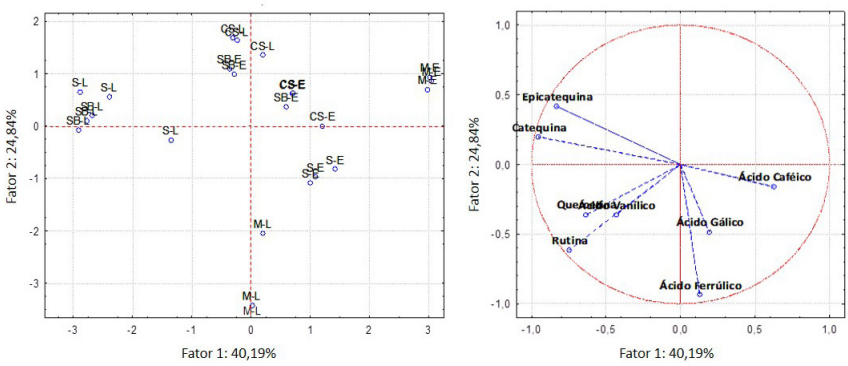

Figura 2. Projeção dos componentes principais das análises de compostos fenólicos para as amostras desidratadas em liofilizador e estufa. CS: Cabernet Sauvignon; S: Sangiovese; SB: Sauvignon Blanc; M: Merlot; E: Estufa; L: Liofilizador.

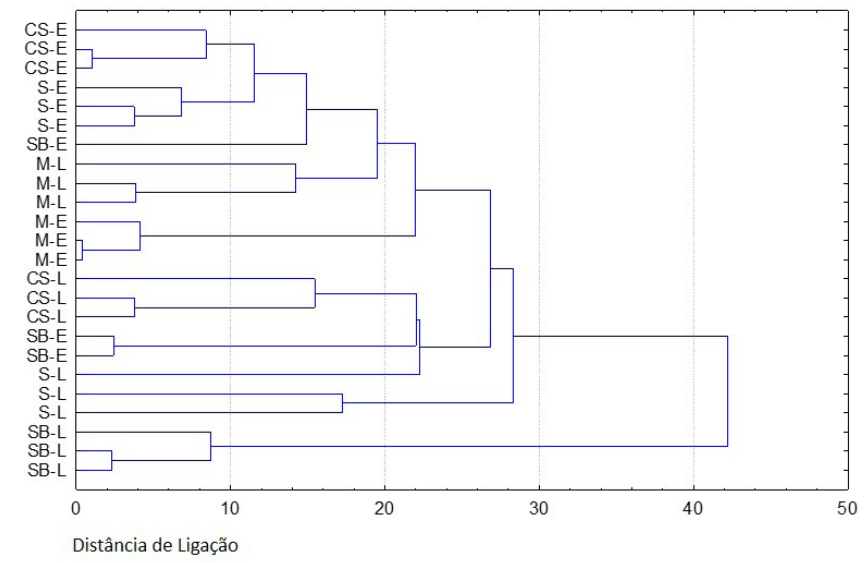

Figura 3. Análise de Agrupamentos Hierárquicos (HCA) para as análises de compostos fenólicos individuais de farinhas de bagaço de uvas submetidas aos tratamentos de desidratação em estufa e em liofilizador. Diagrama para 24 casos apresentados em distâncias Euclidianas. CS: Cabernet Sauvignon; S: Sangiovese; SB: Sauvignon Blanc; M: Merlot; E: Estufa; L: Liofilizador. 
Chamorro et al. (2012) avaliaram o efeito do aquecimento em estufa com circulação de ar sob temperatura de $100^{\circ} \mathrm{C}$ por períodos de 15, 30 e 60 minutos ou em autoclave sobre o conteúdo de compostos fenólicos totais e individuais, antocianinas e atividade antirradicalar pelo método ABTS em farinhas de bagaços de uvas provenientes da vinícola Alcoholeras Reunidas, S.A. (Argamasilla de Alba, Cidade Real, Espanha). Não foram observadas alterações no teor de compostos fenólicos e procianidinas totais quando o bagaço de uva foi submetido ao tratamento térmico em estufa. Para as amostras autoclavadas, observou-se o aumento no teor de ácido gálico (71\%), galocatequina (100\%) e epicatequina galato (129\%). Estes aumentos podem ser justificados pela hidrólise destes compostos a partir das sementes e pelo aumento da disponibilidade dos mesmos (GU et al., 2008).

Para verificar os agrupamentos das amostras em relação à cultivar de uva, foi realizada análise separada (Figura 4) para as amostras liofilizadas, sendo que de 3 a 4 componentes são adequados para explicar
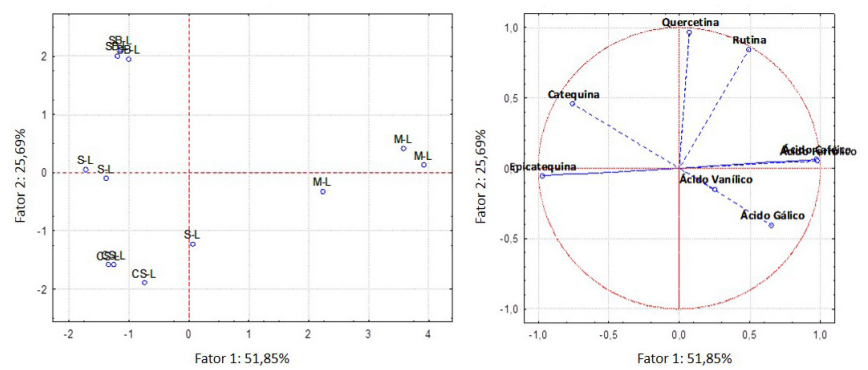

Figura 4. Projeção dos componentes principais das análises de compostos polifenóis para as amostras desidratadas em liofilizador (Guarapuava-PR, 2017). CS-L= Cabernet Sauvignon Liofilizada; SB-L = Sauvignon Blanc Liofilizada; $M-L=$ Merlot Liofilizada; S-L= Sangiovese Liofilizada. mais de $90 \%$ da variabilidade dos teores de compostos fenólicos nas amostras de farinhas liofilizadas.

Segundo o gráfico formado pelos componentes CP1 vs. CP2, as amostras da variedade Merlot se destacam por apresentar maiores teores dos ácidos fenólicos cafeico (4,27 mg $\left.100 \mathrm{~g}^{-1}\right)$, ferrúlico (11,44 mg $\left.100 \mathrm{~g}^{-1}\right)$, gálico (3,82 mg $\left.100 \mathrm{~g}^{-1}\right)$ e vanílico $\left(10,57 \mathrm{mg} 100 \mathrm{~g}^{-1}\right)$ para $77 \%$ das amostras. Observa-se o mesmo, também, de rutina (29,88 mg $\left.100 \mathrm{~g}^{-1}\right)$ e quercetina (35,5 mg $\left.100 \mathrm{~g}^{-1}\right)$, mas teores menores de epicatequina $\left(12,68 \mathrm{mg} 100 \mathrm{~g}^{-1}\right)$ e catequina (26,1 mg $100 \mathrm{~g}^{-1}$ ). A farinha da cultivar Cabernet Sauvignon apresentou menores teores médios de praticamente todos os compostos, com exceção de epicatequina (77,58 mg $\left.100 \mathrm{~g}^{-1}\right)$ e catequina (43,27 mg $\left.100 \mathrm{~g}^{-1}\right)$. A farinha da cv. Sauvignon Blanc apresentou maiores teores médios de quercetina $\left(60,61 \mathrm{mg} 100 \mathrm{~g}^{-1}\right)$, rutina $\left(46,88 \mathrm{mg} 100 \mathrm{~g}^{-1}\right)$ e catequina $\left(72,17 \mathrm{mg} 100 \mathrm{~g}^{-1}\right)$. A cultivar Sangiovese apresentou maiores teores médios de ácido vanílico $\left(18,94 \mathrm{mg} 100 \mathrm{~g}^{-1}\right)$, como é possível observar também na Tabela 1.

No estudo de Abe et al. (2007), com farinhas de bagaços de sete cultivares de videiras produzidas em Minas Gerais, das espécies Vitis labrusca e Vitis vinifera, foi verificado conteúdo superior dos flavanóis catequina - principalmente nas cultivares Niágara Rosada IAC 766 (34,6 mg $100 \mathrm{~g}^{-1}$ ) e Niágara Rosada 196-17 - e epicatequina - nas cultivares Folha de Figo (34,0 mg $\left.100 \mathrm{~g}^{-1}\right)$ e Niágara Rosada IAC 766 (26,0 mg $100 \mathrm{~g}^{-1}$ ).

Bender et al. (2016) avaliaram farinhas de cascas de uvas provenientes de bagaço de uva da cv Marselan (Vitis vinífera) e obtiveram índices de $1,15 \mathrm{mg} \mathrm{g}^{-1}$ para ácido gálico, 0,21 $\mathrm{mg} \mathrm{g}^{-1}$ para ácido cafeico, 2,25 $\mathrm{mg} \mathrm{g}^{-1}$ para quercetina, $1,93 \mathrm{mg} \mathrm{g}^{-1}$ para rutina e $1,10 \mathrm{mg} \mathrm{g}^{-1}$ para catequina.

Tabela 1. Compostos fenólicos em farinhas de resíduos de produção vinícola desidratados em estufa e em liofilizador.

\begin{tabular}{|c|c|c|c|c|c|c|c|c|}
\hline \multicolumn{9}{|c|}{$\begin{array}{l}\text { Compostos Fenólicos } \\
\text { g } 100 \mathrm{~g}^{-1} \\
\text { média } \pm \text { desvio padrão }\end{array}$} \\
\hline & $\begin{array}{l}\text { Merlot } \\
\text { Estufa }\end{array}$ & $\begin{array}{c}\text { Merlot } \\
\text { Liofflizada }\end{array}$ & $\begin{array}{c}\text { Cabernet } \\
\text { Sauvignon } \\
\text { Estufa }\end{array}$ & $\begin{array}{l}\text { Cabernet } \\
\text { Sauvignon } \\
\text { Liofflizada }\end{array}$ & $\begin{array}{c}\text { Sangiovese } \\
\text { Estufa }\end{array}$ & $\begin{array}{l}\text { Sangiovese } \\
\text { Liofilizada }\end{array}$ & $\begin{array}{c}\text { Sauvignon } \\
\text { Blanc } \\
\text { Estufa }\end{array}$ & $\begin{array}{c}\text { Sauvignon } \\
\text { Blanc } \\
\text { Liofilizada }\end{array}$ \\
\hline Ácido Gálico & $2,12 \pm 0,594$ & $3,82 \pm 1,115$ & $3,08 \pm 1,669$ & $4,48 \pm 1,596$ & $1,41 \pm 1,249$ & $1,10 \pm 0,377$ & $1,49 \pm 0,376$ & $2,91 \pm 1,004$ \\
\hline Catequinas & $4,81 \pm 2,342$ & $26,1 \pm 5,513$ & $19,81 \pm 3,809$ & $43,27 \pm 5,108$ & $21,03 \pm 1,278$ & $73,55 \pm 18,829$ & $45,73 \pm 11,52$ & $72,17 \pm 0,936$ \\
\hline Ácido Vanílico & $0,00 \pm 0,00$ & $10,57 \pm 0,639$ & $0,00 \pm 0,00$ & $0,00 \pm 0,00$ & $0,00 \pm 0,00$ & $18,94 \pm 1,434$ & $0,00 \pm 0,00$ & $0,00 \pm 0,00$ \\
\hline Ácido Cafeico & $9,46 \pm 0,349$ & $4,27 \pm 1,457$ & $0,00 \pm 0,00$ & $0,00 \pm 0,00$ & $0,00 \pm 0,00$ & $0,00 \pm 0,00$ & $0,00 \pm 0,00$ & $0,00 \pm 0,00$ \\
\hline Epicatequina & $0,00 \pm 0,00$ & $12,68 \pm 1,53$ & $14,33 \pm 2,175$ & $77,58 \pm 8,79$ & $23,00 \pm 3,63$ & $80,83 \pm 12,996$ & $46,97 \pm 10,967$ & $73,37 \pm 5,08$ \\
\hline Ácido Ferrúlico & $0,00 \pm 0,00$ & $11,44 \pm 2,5$ & $0,00 \pm 0,00$ & $0,00 \pm 0,00$ & $0,00 \pm 0,00$ & $5,71 \pm 1,032$ & $0,00 \pm 0,00$ & $0,00 \pm 0,00$ \\
\hline Rutina & $0,00 \pm 0,00$ & $29,88 \pm 5,477$ & $11,94 \pm 0,497$ & $7,44 \pm 0,277$ & $11,45 \pm 2,382$ & $20,15 \pm 0,497$ & $14,04 \pm 1,005$ & $46,88 \pm 0,915$ \\
\hline Quercetina & $15,85 \pm 0,367$ & $35,5 \pm 2,755$ & $25,13 \pm 1,74$ & $13,95 \pm 0,240$ & $19,795 \pm 1,565$ & $23,84 \pm 0,636$ & $17,11 \pm 0,781$ & $60,61 \pm 1,388$ \\
\hline
\end{tabular}


Makris et al. (2007) analisaram a composição fenólica de bagaços de uva branca, tinta e engaço, e encontraram teores equivalentes em ácido gálico, de 48,26 $\mathrm{mg} \mathrm{g}^{-1}$ para bagaço de uva branca e $54,02 \mathrm{mg} \mathrm{g}^{-1}$ para bagaço de uva tinta. Com base na análise cromatográfica por HPLC da cultivar Bordô (Vitis labrusca), proveniente do processamento vinícola em um vinícola na cidade de Mariópolis, Paraná, Karling et al. (2017) identificaram os compostos ácido gálico, ácido cafeico, ácido p-cumárico e trans-resveratrol, e ácido cafeico.

A Tabela 2 apresenta as correlações entre o método de avaliação de compostos fenólicos totais por Folin-Ciocalteu e compostos fenólicos individuais, confirmando a ideia de que este pode ser considerado um ensaio de poder redutor. A atividade antioxidante dos extratos dos resíduos agroindustriais por esses diferentes métodos de avaliação apresentou uma correlação alta e positiva com o conteúdo de compostos fenólicos totais. Isso sugere que os compostos fenólicos presentes nesses resíduos possuem alta capacidade de sequestro de radicais livres e, por isso, são fontes importantes de agentes antioxidantes primários, de grande importância para a indústria de alimentos (MELO et al., 2011).

Os compostos em uva podem ser divididos em dois grupos: ácidos fenólicos (precursores de flavonoides) e flavonoides (SHI et al., 2003). Sagdic et al. (2011) determinaram o teor de 18 compostos fenólicos pelo método de cromatografia líquida de fase reversa (RP-HPLC) em bagaços liofilizados e extraídos por hexano de cinco cultivares de uva [Emir, Gamay, Kalecik karasi, Narince e Okuzgozu (três vermelhas e duas brancas)] cultivadas na Turquia. Como resultados, dentre os ácidos fenólicos

Tabela 2. Coeficientes de correlação $\left(r^{2}\right)$ entre atividade antioxidante e alguns compostos fenólicos dos extratos dos resíduos agroindustriais.

\begin{tabular}{lcc}
\multicolumn{1}{c}{ Correlação } & $\mathbf{r}^{2}$ & $\boldsymbol{p}$ \\
Cabernet Sauvignon & & \\
Compostos fenólicos totais $\times$ catequina & 1,00 & 0,006 \\
Compostos fenólicos totais $\times$ rutina & 0,994 & 0,049 \\
Compostos fenólicos totais $\times$ quercetina & 0,989 & 0,048 \\
Merlot & & \\
Compostos fenólicos totais $\times$ ácido gálico & 1,00 & 0,003 \\
Compostos fenólicos totais $\times$ ácido vanílico & 0,999 & 0,033 \\
Compostos fenólicos totais $\times$ quercetina & 1,00 & 0,0012 \\
Sangiovese & & \\
ABTS $\times$ HOCl & & \\
ABTS $\times$ ácido gálico & 1,00 & 0,014 \\
ABTS $\times$ rutina & 1,00 & 0,019 \\
ABTS $\times$ quercetina & 1,00 & 0,007 \\
Sauvignon Blanc & & \\
ABTS $\times$ rutina & 1,00 & 0,006 \\
Compostos fenólicos totais $\times$ quercetina & 1,00 & 0,012 \\
\hline
\end{tabular}

avaliados, um dos principais compostos presentes nos extratos foi o ácido gálico, com uma média de valor de

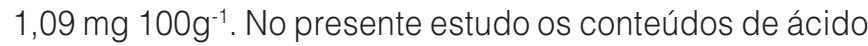
gálico variaram entre 1,42 mg $100 \mathrm{~g}^{-1}$ e 6,04 mg $100 \mathrm{~g}^{-1}$ (cultivares Sangiovese e Cabernet Sauvignon, respectivamente). Entre os flavonoides, catequina foi o principal composto identificado como um valor médio de 508,3 mg $100 \mathrm{~g}^{-1}$, o que vai ao encontro com o presente estudo, no qual catequina também apresentou os mais altos índices, para todas as cultivares avaliadas, com destaque para a cultivar Sauvignon Blanc (45,73 mg $\left.100 \mathrm{~g}^{-1}\right)$. Por outro lado, ácido cumárico, ácido ferrúlico e trans-resveratrol não puderam ser identificados em todos os extratos testados, semelhantemente aos achados neste estudo.

\subsection{Atividade antirradicalar em farinhas de bagaços de uva desidratadas em estufa e liofilizador}

Pelos resultados das análises, ficou evidente que a secagem em estufa reduziu os teores da maioria dos compostos avaliados e, consequentemente, a atividade antioxidante das farinhas de bagaço de uva (Figura 5). Observou-se que o IC50 para o radical ABTS apresentou os melhores resultados, principalmente para as amostras liofilizadas, para as cultivares Merlot $\left(39,52 \mu \mathrm{g} \mathrm{mL}^{-1}\right)$, Cabernet Sauvignon $\left(72,98 \mu \mathrm{g} \mathrm{mL}^{-1}\right)$, Sangiovese $\left(93,18 \mu \mathrm{g} \mathrm{mL}^{-1}\right)$ e Sauvignon Blanc $\left(105,48 \mu \mathrm{g} \mathrm{mL}^{-1}\right)$, nas quais quantidades menores dos bagaços foram capazes de inibir em, pelo

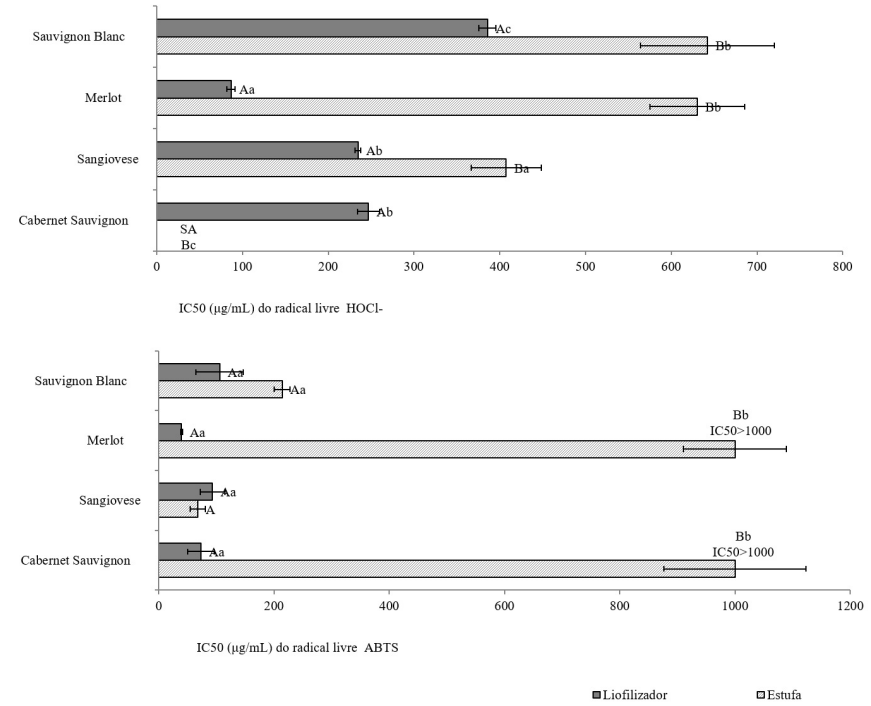

Figura 5. Atividade antioxidante de farinhas de bagaços de uvas desidratadas em estufa e liofilizads, demonstrada pela inibição dos radicais livres ABTS e $\mathrm{HOCl}$ - . 1 SA: sem atividade (não apresentou atividade antioxidante pelo método indicado); IC50>1000: valores superiores à 1000 IC50; Médias seguidas pela mesma letra (maiúsculas para tipo de processo e minúsculas para cultivares) não diferem entre si pelo teste de Student Neuman-Keuls $(p \leq 0,05)$. Barras verticais representam o desvio padrão $(n=3)$. 
menos, $50 \%$ a atividade do radical livre ABTS. Para o IC50 para o radical $\mathrm{HOCl}^{-}$as farinhas da cv. Merlot liofilizada $\left(86,76 \mu \mathrm{g} \mathrm{mL} \mathrm{L}^{-1}\right)$ foram superiores. As farinhas das cultivares Cabernet Sauvignon (246,48 $\mu \mathrm{g} \mathrm{mL} \mathrm{m}^{-1}$ ) e Sangiovese $\left(234,66 \mu \mathrm{g} \mathrm{mL}{ }^{-1}\right)$ não diferiram entre si, mas foram superiores às amostras de Sauvignon Blanc $\left(385,77 \mu \mathrm{g} \mathrm{mL}^{-1}\right)$.

Pela análise multivariada, verificou-se que as amostras com maior atividade antirradicalar foram as liofilizadas e que estas se encontram no eixo negativo da PC1 (Figura 6). Essas amostras também tiveram maiores teores médios de catequina, epicatequina, quercetina, rutina (flavonoides) e ácido vanílico. A atividade antioxidante das farinhas obtidas das diferentes cultivares de uva também apresentou alta correlação positiva com os teores de antocianinas, ácido vanílico e rutina (Tabela 2).

Embora as amostras secas em estufa tenham apresentado maior teor de fenólicos totais, esses compostos não contribuíram para o aumento da atividade antirradicalar. De maneira geral, os compostos determinantes para o aumento desta atividade foram: antocianinas, ácido vanílico, rutina, epicatequina, catequina e quercetina; no entanto, somente a quercetina apresenta correlação positiva para todas as cultivares estudadas, como também a rutina, exceto para a cultivar Merlot (Tabela 2).

A variação da expressão da atividade antirradicalar pelos diferentes métodos sugere que os compostos fenólicos desses resíduos exercem esta atividade por mecanismos de ação distintos, dependendo da polaridade do meio reacional (MELO et al., 2011).

Rockenbach (2008) avaliaram o teor de compostos fenólicos e a atividade antioxidante do bagaço de uvas tintas das variedades Cabernet Sauvignon e Merlot (Vitis vinifera), Bordeaux e Isabel (Vitis labrusca). O bagaço da variedade Cabernet Sauvignon foi o que apresentou o maior teor de compostos fenólicos totais e a maior atividade antioxidante
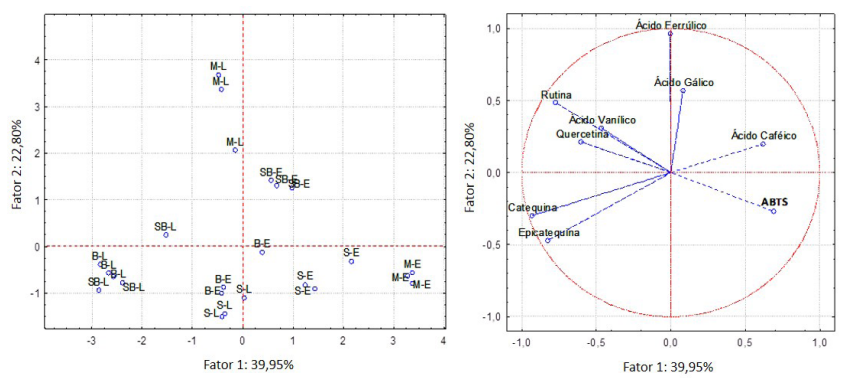

Figura 6. Projeção dos componentes principais das análises de compostos fenólicos e atividade antiradicalar pelo método ABTS + para as amostras desidratadas em estufa e liofilizador (Guarapuava-PR, 2017).
(485,42 $\mu$ mol TEAC g-1 para o método ABTS), resultados superiores aos encontrados neste trabalho.

No estudo de Kallithraka et al. (2005), observou-se correlação estatisticamente insignificante entre antocianinas totais e capacidade antioxidante em cultivares de Vitis vinifera, o que demonstra a importância de outros constituintes da uva. Como exemplo, podem ser citadas catequinas e epicatequinas, que têm demonstrado alta capacidade antioxidante e inibição da proliferação celular (FARIA et al., 2006).

Extratos de bagaços da cultivar Pinot Noir, autoclavados por 15 minutos a $121^{\circ} \mathrm{C}$ e liofilizados, foram transformados em farinhas com diferentes tamanhos de partículas, obtidas por tamisação, e comparados com o bagaço cru. Foi avaliada atividade antirradicalar pelo método IC50 do radical livre ABTS. Os extratos liofilizados não mostraram diferença significativa na atividade antioxidante in vitro entre tratamentos, quando comparados aos bagaços crus. Respostas variáveis foram obtidas no extrato liofilizado, com valores de 8,00 0,62 a 46,60 \pm 2,03 mM de Trolox $100 \mathrm{~g}^{-1}$. Chamorro et al. (2012) avaliaram o efeito do aquecimento em estufa com circulação de ar ou em autoclave, e também não foi observada modificação na atividade antirradicalar entre as amostras.

A comparação entre os processos utilizados para a desidratação das farinhas indica que as farinhas obtidas pela desidratação do bagaço em liofilizador apresentaram melhor atividade antioxidante pelos métodos avaliados. No entanto, embora em menor proporção, as farinhas obtidas pela desidratação do bagaço em estufa das cultivares Sauvignon Blanc e Sangiovese também apresentaram alguma atividade antirradicalar, tornando viável a introdução destes resíduos na alimentação humana, como alternativa de enriquecimento nutricional.

\section{Conclusões}

A comparação entre os processos de desidratação dos bagaços utilizados na produção das farinhas apresentou melhores resultados nos índices de compostos fenólicos totais, individuais, e de antocianinas para as amostras liofilizadas, quando comparadas às farinhas desidratadas em estufa, para todas as cultivares estudadas. Observou-se que catequinas, epicatequinas, quercetina e rutina estiveram entre os compostos fenólicos de maior expressão.

A avaliação da atividade antirradicalar mostrou que as farinhas desidratadas em estufa, sem exceção, tiveram menor capacidade de inibição dos radicais livres, por ambos os métodos de IC50, sendo que a atividade para o radical ABTS apresentou os melhores resultados, principalmente para as amostras liofilizadas, com destaque para a cv. Merlot. Estes resultados reforçam as informações de que as condições de temperatura influenciam na disponibilidade de compostos bioativos nestes alimentos. 
Compostos bioativos e atividade antirradicalar em farinhas de bagaço de uvas de diferentes cultivares desidratadas em liofilizador e em estufa Bennemann, G. D. et al.

\section{Referências}

ABDRABBA, S.; HUSSEIN, S. Chemical composition of pulp, seed and peel of red grape from libya. Global Journal of Scientific Researches. v. 3, n. 2, p. 6-11, 2015.

ABE, L. T.; DA MOTA, R. V.; LAJOLO, F. M.; GENOVESE, M. I. Compostos fenólicos e capacidade antioxidante de cultivares de uvas Vitis labrusca L. e Vitis vinifera L. Ciência e Tecnologia de Alimentos, v. 27, n. 2, p. 394-400, 2007

ABUD, A. K. S.; NARAIN, N. Incorporação da farinha de resíduo do processamento de polpa de fruta em biscoitos: uma alternativa de combate ao desperdício. Brazilian Journal of Food Technology, Campinas, v. 12, n. 4, p. 257-265, 2009. http://dx.doi.org/10.4260/BJFT2009800900020.

AMERICAN PUBLIC HEALTH ASSOCIATION - APHA. Compendium for the microbiological examination of foods. 4th ed. Washington: DOWNES \& ITO, 2001.

BENDER, A. B. B.; LUVIELMO, M. M.; LOUREIRO, B. B.; SPERONI, C. S.; BOLIGON, A. A.; SILVA, L. P., et al. Obtenção e caracterização de farinha de casca de uva e sua utilização em snack extrusado. Brazilian Journal of Food Technology, Campinas, v. 19, 2016. http://dx.doi.org/10.1590/1981-6723.1016.

BRASIL. MINISTÉRIO DA AGRICULTURA, PECUÁRIA E ABASTECIMENTO, SECRETARIA DE POLÍTICA AGRÍCOLA. Projeções do agronegócio, Brasil, 2015/16 a 2025/26. 7. ed. Brasília: SPA/MAPA, 2016. Disponível em: <http://www.agricultura. gov.br/assuntos/politica-agricola/todas-publicacoes-de-politicaagricola/projecoes-do-agronegocio/proj_agronegocio2016.pdf/ view>. Acesso em: 13 set. 2017.

CAMARGo, L. E. A.; PEDROSO, L. S.; Vendrame, S. C.; MAINARDES, R. M.; KHALIL, N. M. Antioxidant and antifungal activities of Camellia sinensis (L.) Kuntze leaves obtained by different forms of production. Brazilian Journal of Biology = Revista Brasileira de Biologia, v. 76, n. 2, p. 428-434, 2016. http://dx.doi.org/10.1590/1519-6984.18814. PMid:26983085.

CATANEO, C. B.; CALIARI, V.; GONZAGA, L. V.; KUSKOSKI. E. M.; FETT, R. Atividade antioxidante e conteúdo fenólico do resíduo agroindustrial da produção de vinho. Semina: Ciências Agrárias, v. 29, p. 93-102, 2008.

CHAMORRO, S.; GOÑI, I.; VIVEROS, A.; HERVERT-HERNÁNDEZ, D.; BRENES, A. Changes in polyphenolic content and antioxidant activity after thermal treatments of grape seed extract and grape pomace. European Food Research and Technology, v. 234, n. 1, p. 147-155, 2012. http://dx.doi.org/10.1007/s00217-011-1621-7.

CORREA-BETANZO, J.; PADMANABHAN, P.; CORREDIG, M.; SUBRAMANIAN, J.; PALIYATH, G. Complex formation of blueberry (Vaccinium angustifolium) anthocyanins during freeze-drying and its influence on their biological activity. Journal of Agricultural and Food Chemistry, v. 63, n. 11, p. 25-63, 2015. http://dx.doi. org/10.1021/acs.jafc.5b00016. PMid:25727778.

DAVIDOV-PARDO, G.; AROZARENA, I.; MARIN-ARROYO, M. R. Stability of polyphenolic extracts from grape seeds after thermal treatments. European Food Research and Technology, v. 232, n. 2, p. 211-220, 2011. http://dx.doi.org/10.1007/s00217-010-1377-5.

FALCÃO, L. D.; DE REVEL, G.; PERELLO, M. C.; MOUTSIOU, A.; ZANUS, M. C.; BORDIGNON-LUIZ, M. T. A survey of seasonal temperatures and vineyard altitude influences on 2-methoxy3-isobutylpyrazine, C13-norisoprenoids, and sensory profile of Brazilian cabernet sauvignon wines. Journal of Agricultural and Food Chemistry, v. 55, n. 9, p. 3605-3612, 2007. http:// dx.doi.org/10.1021/jf070185u. PMid:17394344.

FARIA, A.; CALHAU, C.; DE FREITAS, V.; MATEUS, N. Procyanidins as antioxidants and tumor cell growth modulators. Journal of Agricultural and Food Chemistry, v. 54, n. 6, p. 2392-2397, 2006. http://dx.doi.org/10.1021/jf0526487. PMid:16536624.

FERREIRA, D. F. Sisvar: a computer statistical analysis system. Ciênc. Agrot., v. 35, n. 6, p. 1039-1042, 2011. http://dx.doi. org/10.1590/S1413-70542011000600001.

GIUSTI, M. M.; WROLSTAD, R. E. Anthocyanins: characterization and measurement with uv-visible spectroscopy. In: WROLSTAD, R. E. Current protocols in food analytical chemistry. New York: J. Wiley, 2001. cap. 1, p. 1-13.

GOMEZCACERES, P. L.; OLIVEIRA, F. M. N.; ANDRADE, J. S.; MOREIRA FILHO, M. Cinética de secagem da polpa cupuaçu (Theobroma grandiflorum) pré desidratada por imersão-impregnação. Revista de Ciências Agronômicas, v. 44, n. 1, p. 102-106, 2013. http://dx.doi.org/10.1590/S1806-66902013000100013.

GU, L.; HOUSE, S. E.; ROONEY, L. W.; PRIOR, R. L. Sorghum extrusion increases bioavailability of catechins in weanling pigs. Journal of Agricultural and Food Chemistry, v. 56, n. 4, p. 12831288, 2008. http://dx.doi.org/10.1021/jf072742i. PMid:18251504.

HAMAMA, A. A.; NAWAR, W. Thermal decomposition of some phenolic antioxidants. Journal of Agricultural and Food Chemistry, v. 39, n. 6, p. 1063-1069, 1991.

JACKMAN, R. L.; SMITH, J. L. Anthocyanins and betalains. In: HENDRY, G. A. F.; HOUGHTON, J. D. Natural food colorants. London: Blackie Academic, 1992, p. 183-241.

KALLITHRAKA, S.; MOHDALY, A. A.-A.; MAKRIS, D. P.; KEFALAS, $P$. Determination of major anthocyanin pigments in Hellenic native grape varieties (Vitis vinifera sp.): association with antiradical activity. Journal of Food Composition and Analysis, v. 18, n. 5, p. 375-386, 2005. http://dx.doi.org/10.1016/j.jfca.2004.02.010.

KARLING, M.; BICAS, T. C.; LIMA, V. A.; OLDONI, T. L. C. Grape and apple pomaces from Southern Brazil: valorization of by-products through investigation of their antioxidant potential. Journal of the Brazilian Chemical Society, v. 0, p. 1-9, 2017. http://dx.doi.org/10.21577/0103-5053.20170014.

KARNOPP, A. R.; FIGUEROA, A. M.; LOS, P. R.; TELES, J. C.; SIMÕES, D. R. S.; BARANA, A. C.; KUBIAKI, F. T.; OLIVEIRA, J. G. B.; GRANATO, D. Effects of whole-wheat flour and bordeaux grape pomace (Vitis labrusca L.) on the sensory, physicochemical and functional properties of cookies. Food Science and 
Compostos bioativos e atividade antirradicalar em farinhas de bagaço de uvas de diferentes cultivares desidratadas em liofilizador e em estufa Bennemann, G. D. et al.

Technology, Campinas, v. 35, n. 4, p. 750-756, 2015. http:// dx.doi.org/10.1590/1678-457X.0010.

LARRAURI, J. A.; RUPÉREZ, P.; SAURA-CALIXTO, F. Effect of drying temperature on the stabilitity of polyphenols and antioxidant activity of red grape pomace peels. Journal of Agricultural and Food Chemistry, v. 45, n. 4, p. 1390-1393, Apr. 1997.

MAKRIS, D. P.; BOSKOU, G.; ANDRIKOPOULOS, N. K. Recovery of antioxidant phenolics from white vinification solid by-products employing water/ethanol mixtures. Bioresource Technology, v. 98, n. 15, p. 2963-2967, 2007. http://dx.doi.org/10.1016/j. biortech.2006.10.003. PMid:17110101.

MALACRIDA, C. R.; MOTTA, S. Compostos fenólicos totais e antocianinas em suco de uva. Food Science and Technology, v. 25, n. 4, p. 659-664, 2005. http://dx.doi.org/10.1590/S010120612005000400006.

MANACH, C.; SCALBERT, A.; MORAND, C.; RÉMÉSY, C.; JIMÉNEZ, L. Polyphenols: food sources and bioavailability. The American Journal of Clinical Nutrition, v. 79, n. 5, p. 727-747, 2004.

MARFIL, P. H. M.; SANTOS, E. M.; TELIS, V. R. N. Ascorbic acid degradation kinetics in tomatoes at different drying conditions. Food Science \& Technology, v. 41, n. 9, p. 1642-1647, 2008. http://dx.doi.org/10.1016/j.lwt.2007.11.003.

MELO, P. S.; BERGAMASCHI, K. B.; TIVERON, A. P.; MASSARIOLI, A. P.; OLDONI, T. L. C.; ZANUS, M. C.; PEREIRA, G. E.; ALENCAR, S. M. Composição fenólica e atividade antioxidante de resíduos agroindustriais. Ciência Rural, v. 41, n. 6, p. 1088-1093, 2011.

MOURTZINOS, I.; MAKRIS, D. P.; YANNAKOPOULOU, K.; KALOGEROPOULOS, N.; MICHALI, I.; KARATHANOS, V. T. Thermal stability of anthocyanin extract of Hibiscus sabdariffa $L$. in the presence of $\beta$-Cyclodextrin. Journal of Agricultural and Food Chemisty, v. 56, n. 21, p. 10303-10310, 2008.

MUÑOZ-ESPADA, A. C.; WOOD, K. V.; BORDELON, B.; WATKINS, B. A. Anthocyanin quantification and radical scavenging capacity of Concord, Norton, and Marechal Foch grapes and wines. Journal of Agriculture and Food Chemistry, v. 52, n. 22, p. 6779-6786, 2004.

NARDUZZI, L.; STANSTRUP, J.; MATTIVI, F. Comparing wild american grapes with Itis vinifera: a metabolomics study of grape composition. Journal of Agriculture and Food Chemistry, v. 63, p. 6823-6834, 2015

OLIVEIRA, J.; ALHINHO DA SILVA, M.; TEIXEIRA, N.; DE FREITAS, V.; SALAS, E. Screening of anthocyanins and anthocyaninderived pigments in red wine grape pomace using LC-DAD/ MS and MALDI-TOF techniques. Journal of Agricultural and Food Chemistry, v. 63, n. 35, p. 7636-7644, 2015. http://dx. doi. org/10.1021/acs.jafc.5b00256. PMid:25912410.

PAZ, M. F.; MARQUES, R. V.; SCHUMANN, C.; CORRÊA, L. B.; CORRÊA, É. K. Características tecnológicas de pães elaborados com farelo de arroz desengordurado. Brazilian Journal of Food Technology, Campinas, v. 18, n. 2, p. 128-136, 2015. http:// dx.doi.org/10.1590/1981-6723.6014.
PIOVESANA, A.; BUENO, M. M.; KLAJN, V. M. Elaboração e aceitabilidade de biscoitos enriquecidos com aveia e farinha de bagaço de uva. Brazilian Journal of Food Technology, Campinas, v. 16, n. 1, p. 68-72, 2013. http://dx.doi.org/10.1590/ S1981-67232013005000007.

POUDEL, P. R.; TAMURA, H.; KATAOKA, I.; MOCHIOKA, R. Phenolic compounds and antioxidant activities of skins and seeds of five wild grapes an two hybrids native to Japan. Journal of Food Composition and Analysis, v. 21, p. 622-625, 2008.

ROCKENBACH, I. I. Compostos fenólicos, ácidos graxos e capacidade antioxidante do bagaço da vinificação de uvas tintas (Vitis vinifera e Vitis labrusca). 2008. 112 f. Dissertação (Mestrado em Ciência dos Alimentos)--Universidade Federal de Santa Catarina, Florianópolis, 2008.

SAGDIC, O.; OZTURK, I.; OZKAN, G.; YETIM, H.; EKICI, L.; YILMAZ, M. T. RP-HPLC-DAD analysis of phenolic compounds in pomace extracts from five grape cultivars: evaluation of their antioxidant, antiradical and antifungal activities in orange and apple juices. Food Chemistry, v. 126, n. 4, p. 1749-1758, 2011. http://dx.doi.org/10.1016/j.foodchem.2010.12.075. PMid:25213954.

SEGADE, S. R.; VÁZQUEZ, E. S.; LOSADA, E. D. Influence of ripeness grade on accumulation and extractability of grape skin anthocyanins in different cultivars. Journal of Food Composition and Analysis, v. 21, n. 8, p. 599-607, 2008. http://dx.doi. org/10.1016/j.jfca.2008.04.006.

SHI, J.; YU, J.; POHORLY, J. E.; KAKUDA, Y. Polyphenolics in grape seeds biochemistry and functionality. Journal of Medicinal Food, v. 6, n. 4, p. 291-299, 2003. http://dx.doi. org/10.1089/109662003772519831. PMid:14977436.

SILVA, L. P. Validação de metodologia analítica por CLAE-DAD para determinação de compostos fenólicos e atividade biológica em coprodutos agroindustriais. Dissertação (Programa de Pós-graduação em Tecnologia de Processos Químicos e Bioquímicos)--Universidade Tecnológica Federal do Paraná, Pato Branco, 2016.

SINGLETON, V. L.; ROSSI, J. A. Colorimetry of total phenolics with phosphomolybdicphosphotungstic acid reagents. American Journal of Enology and Viticulture, v. 16, n. 3, p. 144-158, 1965.

SOARES, M.; WELTER, L.; KUSKOSKI, E. M.; GONZAGA, L.; FETT, R. Compostos fenólicos e atividade antioxidante da casca de uvas Niágara e Isabel. Revista Brasileira de Fruticultura, v. 30, n. 1, p. 59-64, 2008. http://dx.doi.org/10.1590/S010029452008000100013.

TABACHNICK, B. G.; FIDELL, L. S. Experimental designs using ANOVA. Plymouth County: Duxbury, 2007.

THE INTERNATIONAL ORGANISATION OF VINE AND WINE OIV. Organisation International de la Vigne et du Vin. 2016. Disponível em: <http://www.oiv.org/uk/accueil/ndex.php>. Acesso em: 15 jul. 2017. 